\title{
Incidencia y características de la esofagitis eosinofílica (EE) en adultos
}

\section{Incidence and characteristics of eosinophilic esophagitis in adults}

\author{
O. Nantes ${ }^{1}$, J.M. Zozaya ${ }^{1}$, F.J. Jiménez-Pérez ${ }^{1}$, J.M. Martínez-Peñuela ${ }^{2}$, F. Borda ${ }^{1}$
}

\section{RESUMEN}

La esofagitis eosinofílica (EE) es una enfermedad caracterizada por la infiltración de la mucosa del esófago por eosinófilos, cuya incidencia en adultos parece estar aumentando en los últimos años, de forma similar a lo que ocurre en otras enfermedades de probable etiología inmunoalérgica. Predomina en varones jóvenes y se manifiesta principalmente por disfagia e impactación alimentaria. Su tratamiento se basa en eliminar el alérgeno potencialmente implicado y la administración de corticoides.

En el presente trabajo se revisan retrospectivamente los casos de EE diagnosticados en el Hospital de Navarra entre enero de 2002 y agosto de 2008, encontrándose 25 pacientes, lo que supone una incidencia de 2,13 casos $/ 10^{5}$ habitantes/año. Un $72 \%$ de nuestros pacientes presentaban disfagia y un $52 \%$ historia de impactación del bolo alimentario, encontrándose alteraciones endoscópicas en 23 de los 25 casos. De 24 pacientes estudiados, un $76 \%$ manifestaban alergia alimentaria o a neumoalérgenos, lo que apoya el fondo inmunoalérgico de la enfermedad y la necesidad de un estudio alergológico en todos las pacientes con EE. La mayoría de nuestros pacientes (22 de 24 valorados) presentaron buena respuesta clínica al tratamiento, que se basó en evitar la exposición al alergeno potencialmente implicado y/o la administración de corticoides (tópicos o sistémicos) y/o la administración de inhibidores de la bomba de protones.

Palabras clave. Esofagitis eosinofílica. Disfagia. Alergia alimentaria. Esófago corrugado.

\begin{abstract}
Eosinophilic esophagitis (EE) is a disease characterised by the infiltration of esophageal mucosa by eosinophils, whose incidence in adults seems to have been increasing in recent years, in a way that is similar to what is occurring with other diseases of a probable immunoallergic aetiology. It predominates in young adults and is mainly expressed by dysphagia and esophageal food impactation. Treatment is based on eliminating the allergen that is potentially involved and the administration of corticoids.

This article offers a retrospective review of $\mathrm{EE}$ cases diagnosed in the Hospital de Navarra between January 2002 and August 2008, with 25 patients found, which represents an incidence of 2.13 cases $/ 10^{5}$ inhabitants/year. Seventy-two percent of our patients showed dysphagia and $52 \%$ a history of food bolus impaction, with endoscopic alterations found in 23 of the 25 cases. Out of 24 patients studied, $76 \%$ showed an alimentary allergy or to neumoallergens, which supports the immunoallergic basis of the disease and the need for an allergy exam in all patients with EE. The majority of our patients (22 out of 24 evaluated) presented a good clinical response to treatment, which was based on avoiding exposure to the potentially involved allergen and/or the administration of corticoids (topical or systemic) and/ or the administration of proton pump inhibitors.
\end{abstract}

Key words. Eosinophilic Esophagitis. Dysphagia. Alimentary allergy. Corrugated esophagus.
1. Servicio de Aparato Digestivo. Hospital de Navarra. Pamplona.

2. Servicio de Anatomía Patológica. Hospital de Navarra. Pamplona.

Recepción el 30 de diciembre de 2008

Aceptación provisional el 12 de febrero de 2009

Aceptación definitiva el 23 de marzo de 2009

\author{
Correspondencia \\ Óscar Nantes Castillejo \\ Irunlarrea, 5-Esc. Centro, $5^{\circ} \mathrm{A}$ \\ 31008 Pamplona \\ E-mail: onantes@yahoo.es
}




\section{INTRODUCCIÓN}

La esofagitis eosinofílica (EE) se caracteriza por una densa infiltración de la mucosa esofágica por eosinófilos, sin que exista afectación del resto del tubo digestivo, y clínica de disfagia e impactación alimentaria en adultos y de enfermedad por reflujo gastroesofágico (ERGE) e intolerancia alimentaria en niños ${ }^{1-3}$. Predomina en varones jóvenes y su incidencia, que en los últimos años parece estar aumentando, se estima en torno a $2,5 / 10^{5}$ habitantes/año en adultos y en $10 / 10^{5}$ en niños ${ }^{1,4,5}$.

Aunque no se han establecido unos criterios diagnósticos definitivos, en la práctica se asume que un recuento de más de 15 eosinófilos por campo de gran aumento (CGA) en la biopsia permite realizar el diagnóstico de EE, siempre en el contexto clínico adecuado y una vez excluidos otros trastornos, en particular la ERGE ${ }^{2}$. En su patogenia interactúan factores genéticos y medioambientales, existiendo cada vez más evidencias que sugieren que se trata de un proceso inflamatorio de etiología inmunoalérgica, determinado por una posible reacción de hipersensibilidad frente a ciertos componentes de la dieta o a neumoalérgenos ${ }^{6-8}$. Por todo ello, actualmente su tratamiento se basa en eliminar la exposición a los posibles alérgenos implicados y en la administración de corticoesteroides por vía tópica.

La mayoría de los pacientes con EE muestran alteraciones en la endoscopia y aproximadamente la mitad alteraciones manométricas, pero ninguna de estas alteraciones ha mostrado clara relación con las manifestaciones clínicas ${ }^{9}$.

El objetivo del presente trabajo es estudiar la incidencia de la EE en la población adulta en nuestro medio, así como sus características clínicas, los hallazgos exploratorios, el tratamiento instaurado y la respuesta al mismo.

\section{MATERIAL Y MÉTODOS}

Estudiamos retrospectivamente las biopsias de esófago de pacientes adultos, en cuya descripción constase el aumento de eosinófilos, realizadas entre el 1 de ene- ro de 2002 y el 31 de agosto de 2008, en el Hospital de Navarra, centro de referencia para una población adulta de 177.714 personas. No se incluyeron en la revisión pacientes pediátricos (menores de 15 años).

Consideramos diagnósticos definitivos de $\mathrm{EE}$ aquellos casos en los que el número de eosinófilos por campo de gran aumento, en la biopsia, era superior a 15 eosinófilos al menos en dos campos (si bien las últimas revisiones consideran suficiente esta cifra en un solo campo), una vez descartados otros trastornos que pudieran justificar la eosinofilia. La presencia de "microabscesos eosinofílicos", de hiperplasia de la capa basal y de inflamación y fibrosis de la lámina propia se consideraron además datos histológicos relevantes que apoyaban el diagnóstico.

Se recogieron los datos epidemiológicos, los síntomas previos al diagnóstico, la historia alergológica, los resultados analíticos y los hallazgos de las exploraciones, así como el tratamiento pautado y la respuesta al mismo.

Este trabajo obtuvo informe favorable del Comité Ético de Investigación Clínica de Navarra en febrero de 2009.

\section{RESULTADOS}

Durante el periodo de estudio, 25 pacientes, 15 varones con una edad media de 30,9 años (rango 17-80) y 10 mujeres con una edad media de 33,9 (rango 19-51), fueron diagnosticados de $\mathrm{EE}$, lo que supone una relación $\mathrm{V} / \mathrm{M}$ de 1,5 y una tasa de incidencia global de 2,13/10 5 hab/año.

Los síntomas más frecuentes en nuestros pacientes fueron la disfagia (72\%) y la impactación alimentaria (52\%), seguidos de la pirosis, el dolor torácico y las náuseas y/o vómitos (Fig. 1).

De los 25 pacientes, 24 fueron estudiados en el Servicio de Alergología presentando 19 algún tipo de diátesis alérgica, lo que supone el $76 \%$ del total de pacientes incluidos. En el estudio alergológico, el $20 \%$ presentaban alergia a algún alimento, el $32 \%$ a neumoalérgenos y el $24 \%$ a ambos (Tabla 1). Quince pacientes (60\%) presen- 
taban eosinofilia periférica $(>7 \%$ del total del recuento leucocitario) y 8 de los 18 pa- cientes a los que se determinó la IgE total la presentaron elevada.

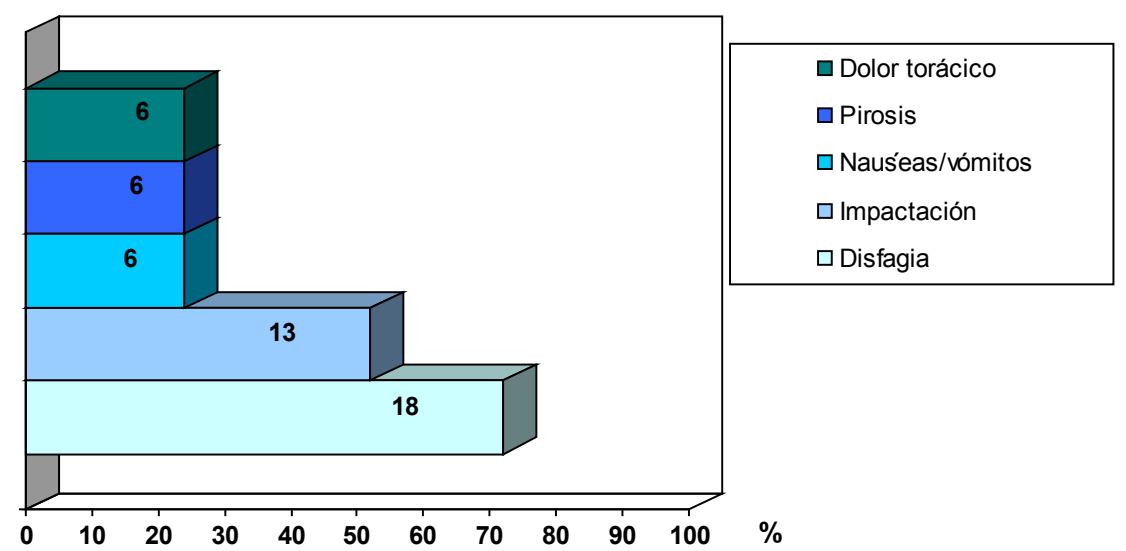

Figura 1. Síntomas en el momento del diagnóstico.

Tabla 1. Tipo de alergias.

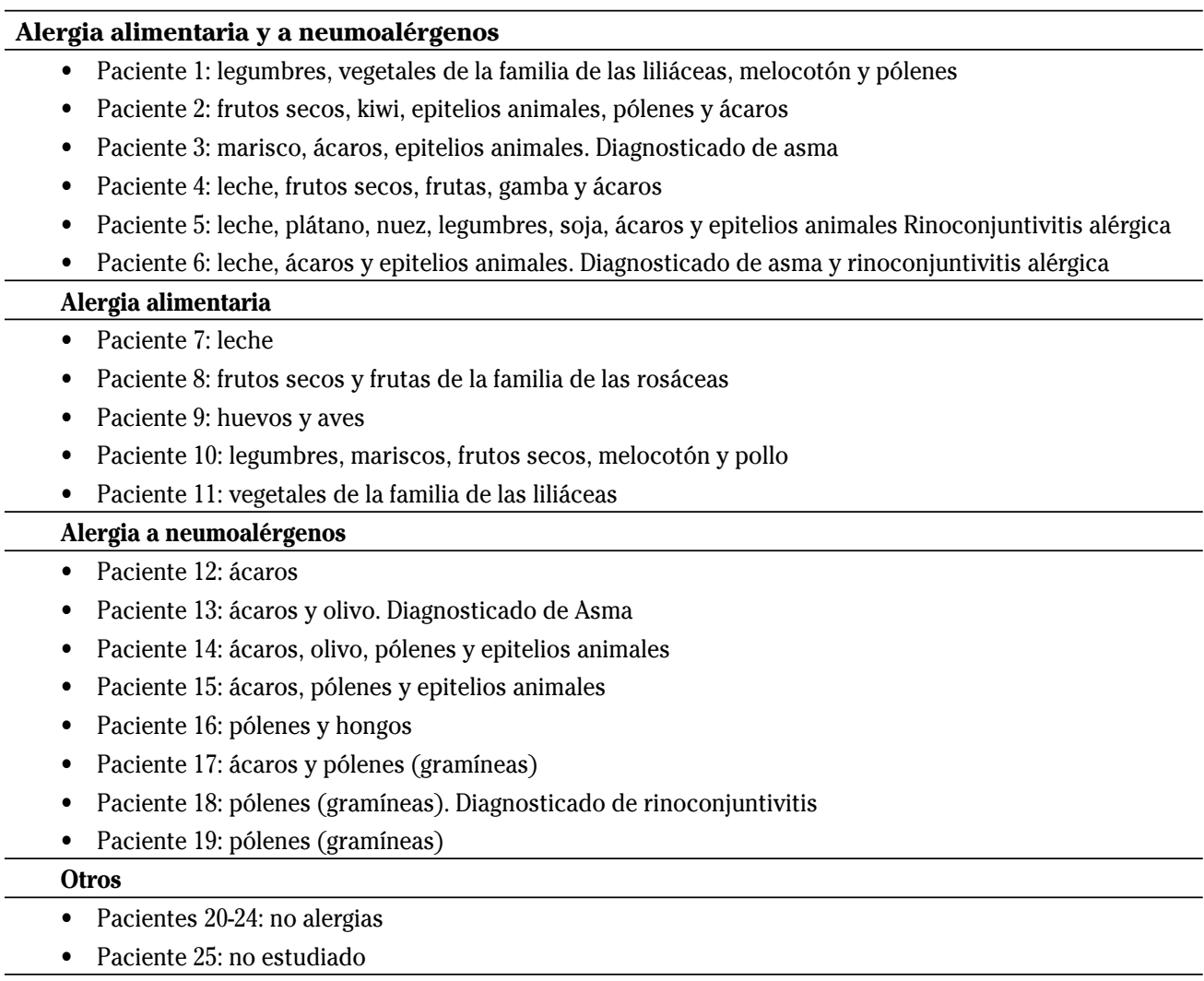


A todos los pacientes se les realizó una endoscopia (Figs. 2 y 3), con toma de biopsias que permitieron el diagnóstico (Figs. 4 y 5); todos los pacientes presentaban $>15$ eosinófilos/CGA al menos en dos campos; en el $80 \%$ se observaron microabscesos eosinofílicos, el $68 \%$ hiperplasia epitelial y el $40 \%$ de los casos en los que la biopsia permitía su valoración (19) fibrosis de la lámina propia. Los hallazgos endoscópicos fueron en el $40 \%$ de los pacientes edema y/o friabilidad de la mucosa, en el $36 \%$ pápulas o exudados blanquecinos, en el $40 \%$ erosiones, objetivándose en el $40 \%$ de los pacientes anillos esofágicos y en el $16 \%$ estenosis. Cinco pacientes (20\%) tenían una endoscopia normal. Dos pacientes presentaron desgarros esofágicos profundos durante la endoscopia (uno de ellos tras la única dilatación realizada en esta serie), presentando ambos buena evolución con tratamiento conservador. A doce pacientes se les realizó una segunda exploración durante el seguimiento, objetivándose en siete de ellos una mejoría endoscópica o histológica con el tratamiento instaurado.

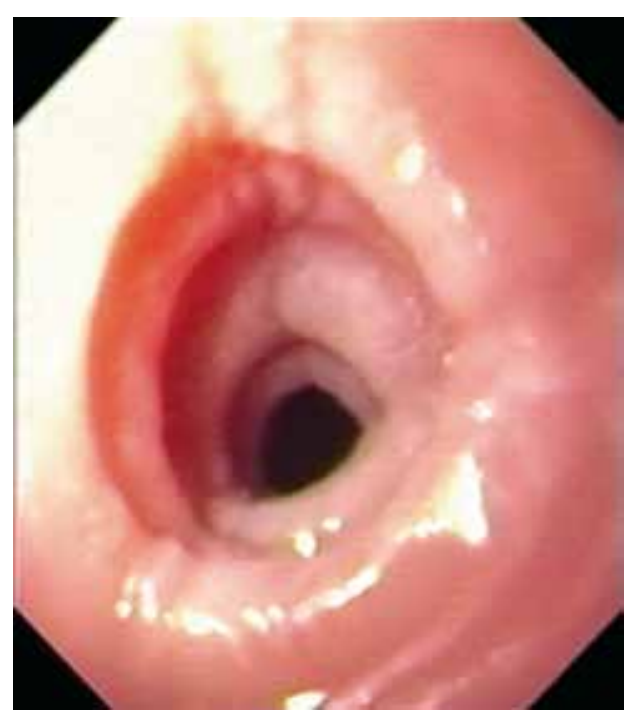

Figura 2. Endoscopia: esófago corrugado, múltiples anillos concéntricos en el esófago que estenosan la luz.

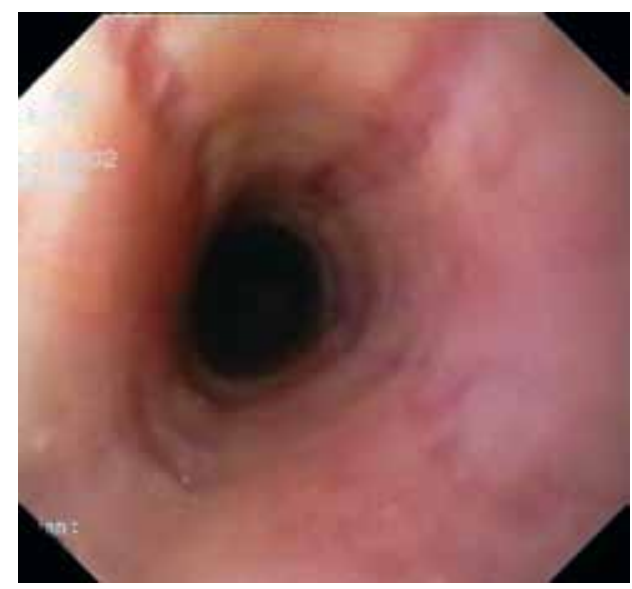

Figura 3. Endoscopia: regueros erosivos lineales en esófago medio.

A nueve pacientes se les practicó un estudio manométrico, detectándose trastornos motores inespecíficos en cuatro, y a nueve una $\mathrm{pH}$-metría, indicada por haber sido etiquetados previamente de ERGE con mala respuesta al tratamiento, siendo normal en todos ellos. En trece pacientes se realizó un estudio baritado gastroduodenal, que mostró una estenosis esofágica en tres de ellos, reflujo gastroesofágico en dos y erosiones en otro.

Todos los pacientes con diátesis alérgicas recibieron instrucciones para evitar la exposición al alergeno potencialmente implicado. Doce pacientes fueron tratados con corticoides, ocho de ellos vía tópica (Tabla 2). Diecisiete pacientes se trataron con inhibidores de la bomba de protones (IBPs), tres de ellos en monoterapia. Un paciente precisó una dilatación endoscópica, por una estenosis no franqueable, tras varios episodios de impactación alimentaria.

De los 24 casos vistos en consultas sucesivas de revisión, 22 refirieron marcada mejoría clínica, y los dos pacientes que continuaban con síntomas presentaron buena respuesta endoscópica y/o histológica al tratamiento. Un paciente llevaba muy poco tiempo de tratamiento como para ser valorada la respuesta al mismo. 
Tabla 2. Tratamiento y respuesta al mismo.

\begin{tabular}{lccc}
\hline & No de pacientes & Respuesta Clínica & $\begin{array}{c}\text { Respuesta histológica } \\
\text { o endoscópica }\end{array}$ \\
\hline EA & 1 & $1 / 1$ & \\
EA + Dilatación & 1 & $1 / 1$ & \\
EA + IBPs & $8^{\#}$ & $6 / 7^{*}$ & $4 / 4$ \\
EA + Corticoides & 6 & $6 / 6$ & $2 / 2$ \\
EA + Corticoides + IBPs & 3 & $2 / 3^{*}$ & $1 / 2$ \\
Corticoides + IBPs & 3 & $3 / 3$ & $0 / 1$ \\
IBPs & $3^{* *}$ & $3 / 3$ & $0 / 3$ \\
\hline
\end{tabular}

EA: evitación del alérgeno.

IBPs: inhibidores de la bomba de protones.

\# Un paciente llevaba poco tiempo de seguimiento como para valorar la respuesta.

* Los dos pacientes que continuaban con clínica durante el seguimiento presentaban mejoría endoscópica y/o histológica.

** De los 3 pacientes tratados sólo con IBPs, en dos se realizó pH-metría que fue normal y en el otro se realizó una segunda gastroscopia tras tratamiento con IBPs, en la que persistía la infiltración por eosinófilos y descartaba la ERGE como responsable de la misma.
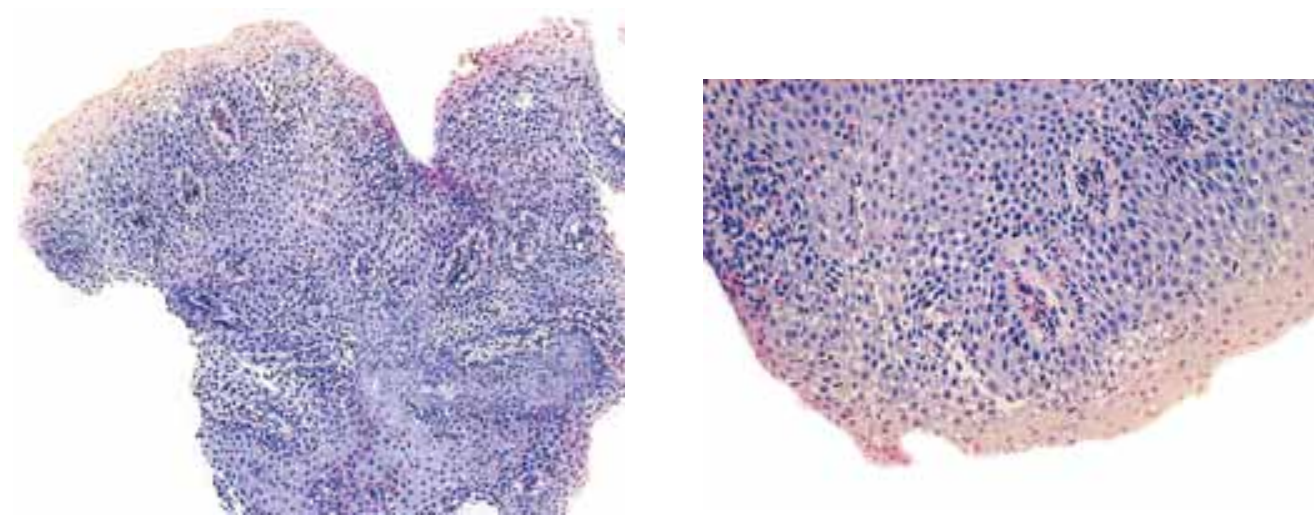

Figuras 4 y 5. Imagen histológica (H-E) que muestra una masiva infiltración por eosinófilos de la mucosa esofágica, formando microabscesos.

\section{DISCUSIÓN}

La EE en adultos, como entidad clínicopatológica independiente, fue descrita en 1993 por Attwood y col ${ }^{10}$. Su trasfondo inmunoalérgico, de manera similar a lo que ocurre en otras enfermedades alérgicas, así como el mejor conocimiento de la entidad por parte de clínicos y patólogos, pueden explicar el incremento en los diagnósticos de EE observado en los últimos años ${ }^{6}$. Estudios recientes estiman la incidencia en adultos en torno a $2,5 / 10^{5}$ habitantes/año ${ }^{1,5}$, cifra similar aunque algo superior a la que hemos encontrado en nuestro estudio (2,13/10 $0^{5}$ hab./año).

Sus manifestaciones clínicas más frecuentes en los adultos, como también se puso de manifiesto en nuestros pacientes, son la disfagia intermitente y la impactación alimentaria ${ }^{1-3}$.

Ya que se ha visto que la mayoría de los pacientes presentan alergia a algún alimento o a algún neumoalergeno y mejoran al evitar la exposición a dichos alergenos, se recomienda que todos los pacientes con $\mathrm{EE}$ sean evaluados por un alergólogo informa- 
do $^{2,11-13}$. En este sentido, el $76 \%$ de nuestros pacientes tenía algún tipo de alergia, especialmente alergia alimentaria o a neumoalergenos. Por otra parte, el $60 \%$ de nuestros pacientes presentaba eosinofilia periférica y el $45 \%$ de los pacientes en los que se determinó la IgE total, la presentaron elevada. Estos resultados son concordantes con los publicados, estimándose que el $50-80 \%$ de los pacientes con EE son atópicos ${ }^{14}$. No obstante, aunque el aumento de eosinófilos en sangre periférica y el aumento de la concentración de IgE total pueden respaldar la presencia de EE y reflejar el grado de afectación tisular, por sí solos no tienen valor diagnóstico ${ }^{2,3}$.

Los hallazgos endoscópicos, en el contexto clínico preciso, resultan muy indicativos de EE. Los más habituales, presentes muchos de ellos en nuestros pacientes, son: friabilidad mucosa con surcos y desgarros longitudinales, edema, pápulas y exudados blanquecinos, pérdida de la elasticidad de la mucosa (mucosa en "papel crêpe"), reducción del calibre, anillos pasajeros o fijos (esófago corrugado) y espasmos ${ }^{1-3}$. Es importante destacar que la mucosa de algunos pacientes con EE puede presentar un aspecto endoscópico normal, por lo que se recomienda tomar biopsias siempre que exista sospecha clínica ${ }^{3}$. Por otra parte, dado que hay una importante variabilidad histológica entre biopsias de un mismo paciente, se aconseja tomar muestras de toda la longitud del esófago para incrementar la sensibilidad diagnóstica ${ }^{15}$.

El criterio básico para el diagnóstico de la EE en todos los estudios publicados ha sido el incremento de la cifra de eosinófilos intraepiteliales, una vez descartadas otras causas de eosinofilia esofágica ${ }^{16,17}$, aunque ni la cifra ni el método han sido uniformes $^{18}$. Actualmente se asume que un recuento de más de 15 eosinófilos/CGA constituye una cifra mínima absoluta para realizar el diagnóstico de EE en el contexto clínico adecuado ${ }^{2}$, recomendándose contabilizar los eosinófilos en los campos de la biopsia que muestran inflamación más intensa. Además se deben considerar otras características, como la presencia de mi- croabscesos eosinofílicos, la distribución superficial de los eosinófilos, la hiperplasia de la capa basal, la elongación papilar, la inflamación y fibrosis de la lámina propia y la degranulación de los eosinófilos ${ }^{19}$, que sin ser patognomónicas pueden apoyar el diagnóstico, siendo especialmente relevantes entre nuestros pacientes la presencia de microabscesos eosinofílicos (80\%).

La respuesta clínica a los IBPs no permite diferenciar entre EE y ERGE, ya que aunque los IBPs no son el tratamiento principal de la EE, pueden inducir mejoría sintomática, especulándose que el esófago de los pacientes con EE es más sensible al ácido, por lo que los IBPs pueden utilizarse como tratamiento adicional en la EE $E^{2,9,20}$. Por ello, cuando la distinción entre EE y ERGE no es evidente, a pesar de la endoscopia y/o la histología, debe realizarse una $\mathrm{pH}$-metría para descartar reflujo patológico como causa de la eosinofilia esofágica. Por otra parte, debe considerarse el diagnóstico de EE en pacientes con clínica de ERGE con mala respuesta a IBPs y que presentan $\mathrm{pH}$ metría normal.

La manometría no ha demostrado valor diagnóstico en los pacientes con EE, encontrándose alteraciones inespecíficas, fundamentalmente de tipo hipomotilidad, en aproximadamente el $50 \%$ de los adultos con EE (en nuestro estudio en 4 de los 9 pacientes a los que se les practicó una manometría). Además, las alteraciones motoras encontradas (hipo o hipermotilidad), no han mostrado clara correlación con las manifestaciones clínicas de los pacientes ${ }^{9}$. El tránsito gastroduodenal puede ser útil en pacientes con disfagia para descartar anomalías anatómicas y/o en pacientes con estenosis, para completar el estudio ${ }^{2}$.

El tratamiento debe dirigirse a aliviar los síntomas y, de manera ideal, a conseguir la remisión de la eosinofilia esofágica y evitar la progresión a una disfunción esofágica crónica. En la mayoría de los pacientes, la eliminación de los antígenos alimentarios sirve para tratar con éxito tanto los síntomas como las anomalías histopatológicas subyacentes. En el caso de pacientes pediátricos la eliminación de 
ciertos antígenos o dietas de leche maternizada elemental, ha demostrado ser un tratamiento eficaz. En el caso de los adultos, siguen siendo necesarios más estudios para evaluar el tratamiento alimentario ya que las pruebas alergológicas pueden resultar insuficientes para la detección de los agentes etiológicos responsables ${ }^{21,22}$. Por el momento no se recomienda la realización de pruebas de alergia alimentaria in vitro y para las pruebas alimentarias empíricas, la combinación de pruebas de punción y de parche han arrojado resultados prometedores.

Los corticoides sistémicos y tópicos inducen la remisión de las manifestaciones anatomoclínicas agudas de la EE, pero la enfermedad tiende a reaparecer cuando se retiran. Los corticoides sistémicos, debido a sus efectos secundarios, se reservan para los casos severos. La administración de corticoides por vía tópica oral (fluocortisona o budesonida) durante 6-8 semanas ofrece diversas ventajas (dosis menor, rápida metabolización hepática y acción directa sobre la mucosa esofágica) ${ }^{1,2,6}$. Aunque pueden inducir la aparición de micosis localizadas, varios estudios han documentado su seguridad en tratamientos cortos, estando pendiente su evaluación como tratamiento de mantenimiento.

La dilatación del esófago es útil para los pacientes que presentan estenosis sintomáticas que producen impactación del bolo alimentario. Sin embargo, en vista del riesgo de desgarro y perforación, antes de la dilatación siempre debe plantearse el tratamiento farmacológico o instaurar un régimen alimentario especial ${ }^{2}$. Ya hemos señalado que el único paciente de nuestra serie que precisó una dilatación sufrió un profundo desgarro esofágico, que precisó hospitalización.

Respecto a los estabilizadores de los mastocitos, el cromoglicato disódico no parece tener utilidad terapéutica. Los antagonistas del receptor de los leucotrienos producen alivio sintomático cuando se administran en dosis elevadas, pero no han demostrado efecto alguno sobre la eosinofilia esofágica. Los estudios publicados no avalan el uso de estos medicamentos como tratamiento de la EE. En el futuro es posible que los anticuerpos monoclonales, como los anti-IL-5, brinden posibilidades de tratamiento ${ }^{2}$.

Durante el tratamiento se aconseja la realización frecuente de consultas de seguimiento para valorar la evolución sintomática, la adherencia al tratamiento y los efectos adversos. Sin embargo, hay pocos estudios que orienten el intervalo óptimo de observación y controles endoscópicos, y se debe tener presente que las endoscopias de control también se asocian a posibles complicaciones ${ }^{23-25}$.

Como conclusión, queremos señalar que en nuestro medio se observa una incidencia de EE de 2,13/105 hab./año, similar a la descrita. La disfagia y la impactación alimentaria son las manifestaciones más frecuentes y, por tanto, la EE debe ser considerada en el diagnóstico diferencial de pacientes con estos síntomas. Dada la elevada frecuencia de diátesis alérgicas entre nuestros pacientes (el 76\% tiene historia alérgica positiva y más del $50 \%$ eosinofilia periférica y/o elevación de IgE), consideramos que todo paciente con EE debe ser valorado en alergología y que la evitación de la exposición a los alergenos debe ser la base del tratamiento.

\section{BIBLIOGRAFÍA}

1. YAN BM, ShafFer EA. Eosinophilic esophagitis: A newly established cause of dysphagia. World J Gastroenterol 2006; 12: 2328-2334.

2. Furuta GT, Liacouras CA, Collins MH, Gupta SK, Justinich C, Putnam PE et al. Eosinophilic esophagitis in children and adults: a systematic review and consensos recomendations for diagnosis and treatment. Gastroenterology 2007; 133: 1342-1363.

3. Sgouros SN, Vergele C, Mantides A. Eosinophilic esophagitis in adults: a systematic review. Eur J Gastoenterol Hepatol 2006; 18: 211-217

4. Noel RJ, Putnam PE, Rothemberg ME. Eosinophilic esophagitis. N Engl Med 2004; 351: 940-941.

5. Croese J, Fairley SK, Masson JW, Chong AK, WhitaKer DA, KANOWSKI PA et al. Clinical and endoscopic features of eosinophilic esophagitis in adults. Gastrointest Endosc 2003; 58: 516-522. 
6. LucEndo Villarín AJ, De Rezende L. Eosinophilic esophagitis. Review of current clinical and physiopathological concepts. Gastroenterol Hepatol 2007; 30: 234-243.

7. Blanchard C, Rothemberg ME. Basics patogenesis of eosinophilic esophagitis. Gastrointest Endosc Clin N Am 2008; 18: 133-143.

8. Lucendo AJ, Navarro M, Comas C, Pascual JM, Burgos E, Santamaría L et al. Inmunophenotypic characterization and quantification of the epithelial inflammatory infiltrate in eosinophilic esophagitis through stereology: an analisis of the cellular mechanisms of the disease and the immunologic capacity of the esophagus. Am J Surg Pathol 2007; 31: 598-606.

9. Martín Martín L, Santander Vaquero C, Sánchez Prudencio S, Canteo Perona J, Gisbert JP, MoreNo Otero R. Eosinophilic esophagitis in the adult: clinical, endoscopic, pH-metric, and manometric findings. Rev Esp Enferm Dig 2008; 100: 476-480.

10. Attwood SE, Smyrk TC, Demeester TR, Jones JB. Esophageal eosinophilia with dysphagia. A distinct clinicopathologic syndrome. Dig Dis Sci 1993; 38: 109-116.

11. Kagalwalla AF, Sentongo TA, Ritz S, Hess T, NELSON SP, EMERICK KM et al. Effect of six-food elimination diet on clinical and histologic outcomes in eosinophilic esophagitis. Clin Gastroenterol Hepatol 2006; 4: 1097-1102.

12. Markowitz JE, Spergel JM, Ruchelli E, Liacouras CA. Elemental diet is an effective treatment for eosinophilic esophagitis in children and adolescents. Am J Gastroenterol 2003; 98: 777-782.

13. De Angelis P, Markowitz JE, Torroni F, Caldaro T, Pane A, Morino G et al. Paediatric eosinophilic oesophagitis: towards early diagnosis and best treatment. Dig Liver Dis 2006; 38: 245-251.

14. Liacouras CA, Spergel JM, Ruchelli E, Verma R, Mascarenhas M, Semeao E et al. Eosinophilic esophagitis: a 10-years experience in 381 children. Clin Gastroenterol Hepatol 2005; 3 : 1198-1216.

15. Gonsalves N, Policarpo-Nicolas M, Zhang Q, Rao MS, HiRANO I. Histopatologic variabilitiy and endoscopic correlates in adults with eosinophilic esophagitis. Gastrointest Endosc 2006; 64: 313-319.
16. Kaye PV, Majumdar D, Ragunath K, Aithal G. Beware the eosinophils. Histopathology 2007; 50: 936-938.

17. Rodrigo S, Abboud G, Oh D, DeMeester SR, Hagen J, Lipham J et al, Chandrasoma P. High intraepithelial eosinophil counts in esophageal squamous epithelium are not specific for eosinophilic esophagitis in adults. Am J Gastroenterol 2008; 103: 435-442.

18. Dellon ES, Aderoju A, Woosley JT, Sandler RS, SHAHEEN NJ. Variability in diagnostic criteria for eosinophilic esophagitis: a systematic review. Am J Gastoenterol 2007; 102: 2300-2313.

19. Parfitt JR, Gregor JC, Suskin NG, JaWA HA, DriMAN DK. Eosinophilic esophagitis in adults: distinguishing features from gastroesophageal reflux disease: a study of 41 patients. Mod Pathol 2006; 19: 90-96.

20. Ngo P, Furuta GT, Antonioli DA, Fox VL. Eosinophils in the esophagus-peptic or allergic EE? Case series of three patiens with esophageal esosinophia. Am J Gastroenterol 2006; 101: 1666-1670.

21. Spergel JM, Andrews T, Brown-Whitehorn TF, Beausoleil JL, Liacouras CA. Treatment of eosinophilic esophagitis with specific food elimination diet directed by a combination of skin prick and patch tests. Ann Allergy Asthma Immunol 2005; 95: 336-343.

22. Spergel JM, Mascarenhas M, Liacouras CA. The uses of skin prick tests and patch tests to identify causative foods in eosinophiclic esophagitis. J Allergy Clin Immunol 2002; 109: 363-368.

23. Straumann A, Bussmann C, Zuber M, Vannini S, Simon HU, Schoepfer A. Eosinophilic esophagitis: analysis of food impaction and perforation in 251 adolescent and adult patients Clin Gastroenterol Hepatol 2008; 6: 598-600.

24. Robles-Medranda C, Villard F, Bouvier R, DumorTIER J, LACHAUX A. Spontaneous esophageal perforation in eosinophilic esophagitis in children. Endoscopy 2008; 40: E171.

25. Cohen MS, Kaufman AB, Palazzo JP, Nevin D, DiMARINO AJ JR, COHEN S. An audit of endoscopic complications in adult eosinophilic esophagitis. Clin Gastroenterol Hepatol 2007; 5: 1149-1153. 